hep-lat/0110143

October 2001

\title{
Hadron Spectrum for Quenched Domain-Wall Fermions with DBW2 Gauge Action*
}

\author{
Yasumichi Aoki ${ }^{\mathrm{a}}[\mathrm{RBC} \text { collaboration }]^{\dagger}$ \\ ${ }^{a}$ RIKEN BNL Research Center, Brookhaven National Laboratory, Upton, NY 11973, USA
}

We investigate basic physical quantities for quenched simulation with domain-wall fermions and the DBW2 gauge action. Masses and decay constant of pseudoscalar mesons are measured. Scaling properties are tested.

\section{INTRODUCTION}

Domain wall fermion (DWF) [1.2] is a promising tool to calculate hadronic quantities where chiral symmetry is important. Precise calculation of kaon mixing parameter $B_{K}$ is one success of this formulation [3]. More challenging calculations [4, 5] of kaon weak matrix element for $\epsilon^{\prime} / \epsilon$ show a first reliable determination within lowest order chiral perturbation theory. Attempt to calculate nucleon matrix elements is also underway [6].

The good chiral property of DWF comes at an obvious cost, which is a substantial increase of computational effort associated with the size of the fifth dimension $L_{s}$. This makes taking the continuum limit by performing lattice finer than $a^{-1} \simeq 2 \mathrm{GeV}$ difficult. Going to a coarser lattice to have another point for the continuum extrapolation could be a better strategy. However, results [7] 8] on coarser lattices show that an incredibly large size for the fifth dimension is required to keep the residual chiral symmetry breaking small.

Motivated by the fact that improving the gauge action reduces the residual chiral symmetry breaking at finite $L_{s}$ significantly at $a^{-1} \simeq 2$ $\mathrm{GeV}[7]$, we found the DBW2 action [9], which is a non-perturbatively determined Iwasaki-type action[10], gave much more improvement for the chiral properties 11, 12]. Here we investigate basic physical quantities for the DBW2 gauge action for quenched DWF to see the viability of this approach down to $a^{-1} \simeq 1.3 \mathrm{GeV}$.

\footnotetext{
*Talk presented at Lattice 2001, Berlin, Germany.

${ }^{\dagger}$ We thank RIKEN, Brookhaven National Laboratory and the U.S. Department of Energy for providing the facilities essential for the completion of this work.
}

Table 1

Simulation parameters and calibration.

\begin{tabular}{cccccc}
\hline$\beta$ & $M_{5}$ & $L_{s}$ & stat. & $m_{\text {res }} a$ & $a_{\rho}^{-1}(\mathrm{GeV})$ \\
\hline 0.87 & 1.8 & 16 & 100 & $5.7(3) 10^{-4}$ & $1.28(3)$ \\
1.04 & 1.7 & 16 & 200 & $1.7(1) 10^{-5}$ & $1.97(4)$ \\
\hline
\end{tabular}

We perform the simulation on a $16^{3} \times 32$ lattice with DBW2 action at $\beta=0.87$ and 1.04 in Iwasaki type parameterization, which corresponds to $a^{-1} \simeq 1.3$ and $2 \mathrm{GeV}$ respectively. The domain wall hight $M_{5}$ is tuned in the first decimal place so that it minimizes the residual quark mass $m_{\text {res }}$, although it is not necessary that it be done to this accuracy.

\section{RESULTS FOR $a^{-1} \simeq 1.3 \mathrm{GeV}$}

The residual quark mass $m_{\text {res }}$ is defined through the ratio

$R(t)=\left\langle J_{5 q}(t) P(t)\right\rangle /\langle P(t) P(0)\rangle$

at $t \gg a$, where $J_{5 q}$ represents explicit breaking of chiral symmetry in the the divergence of "conserved current" $\mathcal{A}_{\mu}$, and $P$ is the pseudoscalar density operator. We list the value of $m_{r e s}$ in Table 1. For $a^{-1} \simeq 1.3 \mathrm{GeV}$ unrenormalized value of $m_{\text {res }} \simeq 0.7 \mathrm{MeV}$, which is smaller than that obtained for the Wilson gauge action at finer lattice, $a^{-1} \simeq 2 \mathrm{GeV}$ and the same $L_{s}, m_{\text {res }} \simeq 2.4$ $\mathrm{MeV}[\mathbb{8}]$.

The pion mass can be extracted from a two point function constructed from pion interpolating operators. We consider two different twopoint functions. One is the pseudoscalar - pseudoscalar, $\langle P P\rangle$, the other is the local axial - axial correlator, $\left\langle A_{0} A_{0}\right\rangle$. The former has a $1 / \mathrm{m}^{2}$ 


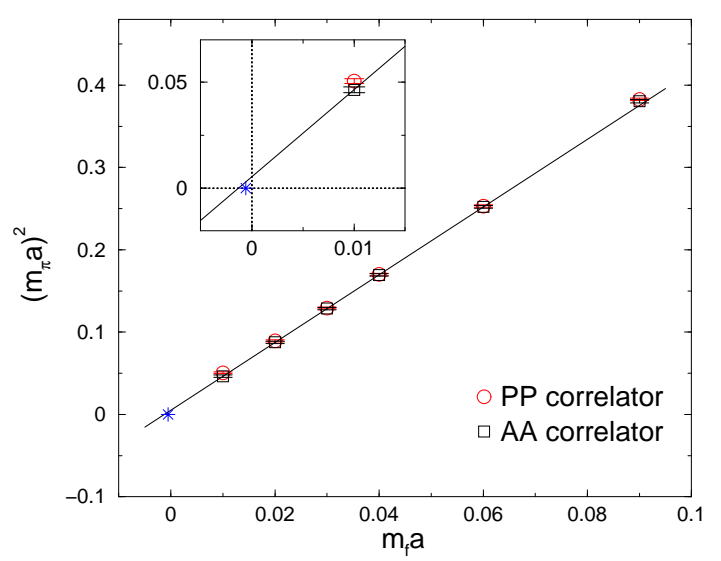

Figure 1. Pion mass squared as a function of quark mass for $a^{-1} \simeq 1.3 \mathrm{GeV}$. Asterisk indicates the point $m_{f}=-m_{\text {res }}$.

and $1 / m$ pole from zero modes in finite volume, while the later has only $1 / m$. Figure 11 shows the pion masses from those different two point functions are consistent with each other down to $m_{f}=0.02$. The difference begins to show up at the lightest mass $m_{f} a=0.01$, however it is quite small compared to the value of mass itself.

A linear fit of the $\left\langle A_{0} A_{0}\right\rangle$ correlator for $0.01 \leq$ $m_{f} \leq 0.06$ is quite good $\left(\chi^{2} / \operatorname{dof}=0.03 / 3\right)$. However, $\mathrm{x}$-intercept is $m_{f}=-1.3(4) 10^{-3}$, so it overshoots $m_{f}=-m_{r e s}$, where the pion mass should vanish. This location of the intercept is guaranteed with accuracy of $O\left(a^{2}\right)$ by the WardTakahashi identity when all possible finite size effects have been removed 8 . With this constraint one can fit to a quenched chiral log formulae,

$$
\begin{aligned}
\left(m_{\pi} a\right)^{2} & =a_{0} m\left(1+a_{1} \log m\right)+a_{2} m^{2} \\
m & \equiv m_{f} a+m_{r e s} a .
\end{aligned}
$$

Results are listed in the Table 2. Both fits with or without quadratic term give reasonable value of $a_{1}$.

Table 2

Fit result of pion mass. "_" indicates that the parameter is constrained to be zero.

\begin{tabular}{ccccc}
\hline$m_{f}$ & $a_{0}$ & $a_{1}$ & $a_{2}$ & $\chi^{2} /$ dof \\
\hline $0.01-0.06$ & $3.9(2)$ & $0.03(2)$ & - & $0.5 / 3$ \\
$0.01-0.09$ & $3.1(5)$ & $0.09(5)$ & $4.9(23)$ & $0.04 / 3$ \\
\hline
\end{tabular}

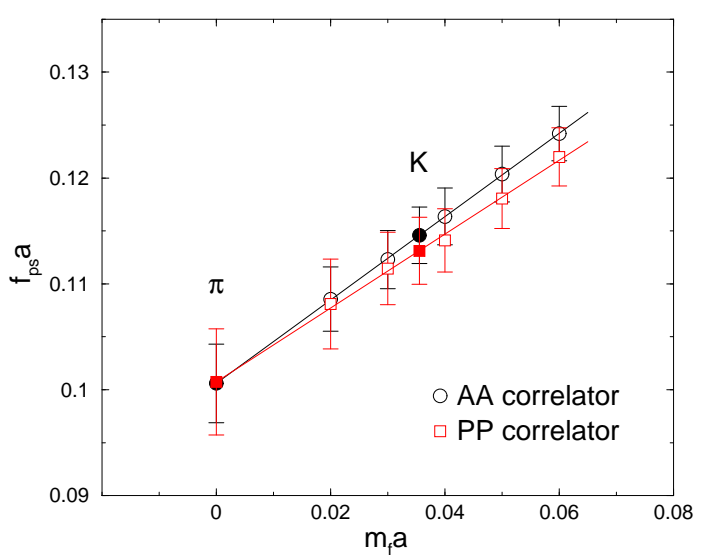

Figure 2. Pseudoscalar decay constant as a function of quark mass at $a^{-1} \simeq 1.3 \mathrm{GeV}$.

We determine the pseudoscalar decay constant using two methods [8]. One is an estimate from the $\left\langle A_{0} A_{0}\right\rangle$ correlator with the renormalization factor $Z_{A}$ nonperturbatively determined from the ratio of $\left\langle\mathcal{A}_{0} P\right\rangle$ and $\left\langle A_{0} P\right\rangle$. The other uses the $\langle P P\rangle$ correlator with the equation $\Delta_{\mu} \mathcal{A}_{\mu} \simeq$ $2\left(m_{f}+m_{\text {res }}\right) P$ from the Ward-Takahashi identity. As seen in the Figure 2 the two methods give consistent results, indicating the effect of chiral symmetry breaking can be absorbed in the shift of $m_{f}$. Also the possible zero mode effects, which influence the two correlators differently, appear to be small, which is a confirmation of what we saw for the pion mass down to $m_{f}=0.02$.

\section{SCALING PROPERTIES}

Figure 3 plots the rho meson mass extrapolated to the chiral limit as a function of lattice spacing. Both axes are normalized by $r_{0}$ calculated from the heavy quark potential. Together with the data of this work, results with Wilson gauge action are shown. Those data show that the scales determined by different methods agree very well for both the DBW2 and the Wilson gauge action for DWF. This good scaling is also seen in the nucleon mass and $K^{*}$ mass normalized by rho meson mass. This also holds when one chooses $r_{0}$ for the input of scale, which is obvious from above discussion.

Scaling of the pseudoscalar decay constant 


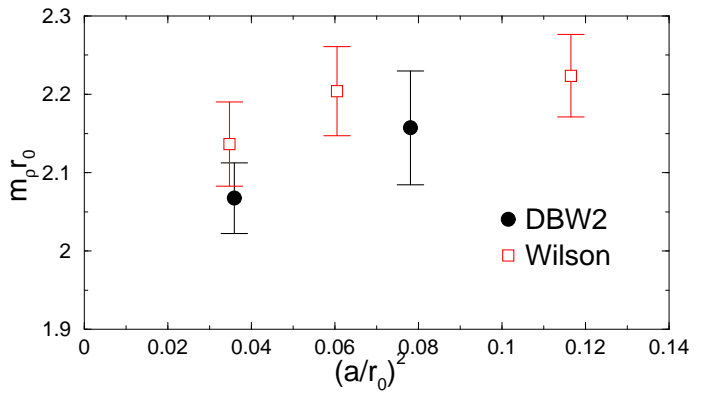

Figure 3. Consistency check of scales from the rho meson mass and the heavy quark potential.

(Fig. 5) is also good. $f_{\pi}$ is in good agreement with the experimental value. $f_{K}$ appears to be smaller than the experimental value, which could be caused by the effects of quenching 13 .

\section{CONCLUSION}

We have attempted to use the DBW2 gauge action in a quenched domain wall fermion simulation. Good chiral properties for $a^{-1} \simeq 1.3 \mathrm{GeV}$ has been observed. We have investigated scaling of hadronic observables, which also turned out to be good. We have also confirmed that the values were consistent with those obtained with the Wilson gauge action.

The statistics presented here is not sufficient for the precision measurement for those observables calculated here. However, we can argue that there is no evidence for any difficulty with this choice of gauge action, rather it has lots of good features.

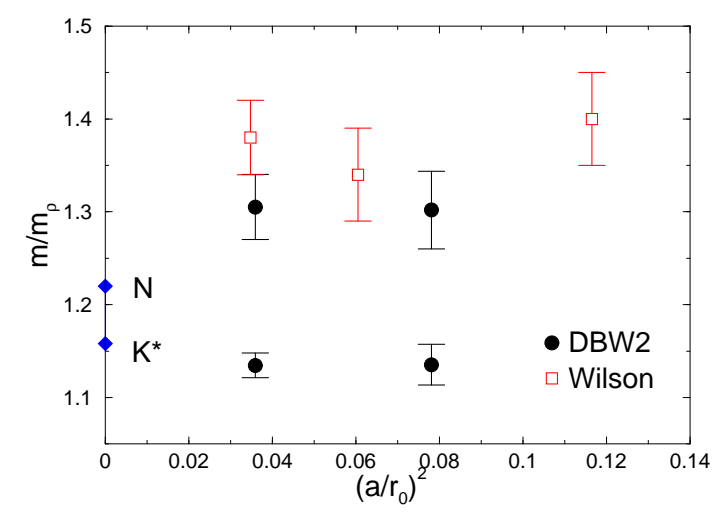

Figure 4. Scaling plot of nucleon and $K^{*}$ mass. Diamonds are experimental values.

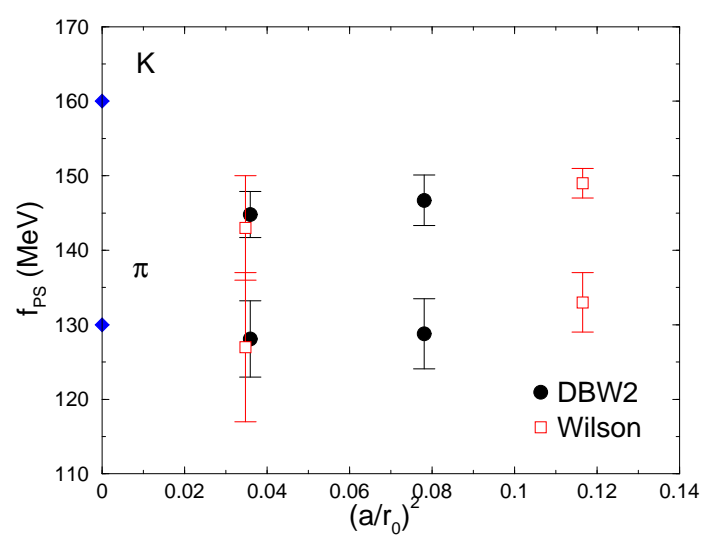

Figure 5. Scaling plot of pseudoscalar decay constant. Diamonds are experimental values.

An important benefit from the use of this gauge action is that residual chiral symmetry breaking is quite small even for a coarse lattice with $a^{-1} \simeq 1.3 \mathrm{GeV}$. Therefore it may be suitable for demanding calculation like $\epsilon^{\prime} / \epsilon$. Once we have the results for $a^{-1} \simeq 1.3 \mathrm{GeV}$ and finer lattices, we will be able to discuss the scaling property for those quantities.

\section{REFERENCES}

1. D. Kaplan, Phys. Lett. B288 (1992) 342-347.

2. V. Furman, Y. Shamir, Nucl. Phys. B439 (1995) 54 .

3. T. Blum and A. Soni, Phys. Rev. Lett. 79 (1997) 3595; CP-PACS collaboration: A. Ali Khan et al., hep-lat/0105020.

4. CP-PACS collaboration: J. Noaki et al., heplat/0108013.

5. T. Blum et al., hep-lat/0110075.

6. S. Sasaki et al., these proceedings.

7. CP-PACS collaboration: A. Ali Khan et al., Phys. Rev. D63 (2001) 114504.

8. T. Blum et al., hep-lat/0007038.

9. T. Takaishi, Phys. Rev. D54 (1996) 1050; P. de Forcrand et al., Nucl. Phys. B577 (2000) 263.

10. Y. Iwasaki, UTHEP-118 (1983).

11. K. Orginos, these proceedings.

12. T. Izubuchi, these proceedings.

13. C. Bernard, M. Golterman, Phys. Rev. D46 (1992)853. 